\title{
The Study of Complementary Application Between Grammar Translation Method and Cognitive Approach in College English Translation Teaching
}

\author{
Wang Ke*, Wu Jiaping \\ Foreign Language Department, North China Electric Power University, Beijing, China
}

\section{Email address}

1125865328@qq.com (Wang Ke), jpingwu@126.com (Wu Jiaping)

*Corresponding author

\section{To cite this article:}

Wang Ke, Wu Jiaping. The Study of Complementary Application Between Grammar Translation Method and Cognitive Approach in College English Translation Teaching. Science Innovation. Vol. 5, No. 5, 2017, pp. 263-266. doi: 10.11648/j.si.20170505.13

Received: May 24, 2017; Accepted: June 27, 2017; Published: July 19, 2017

\begin{abstract}
With the expansion of China's contacts with foreign countries, the cultivation of foreign language translation talents has become particularly urgent. As a bridge of cooperation and communication, translation plays an important role in the cooperation between China and other countries in the world. However, at present, our country still lacks a lot of high level translators. As the backbone of high-quality talents, college students are also the reserve forces for the cultivation of national translators. Therefore, it is very important to improve college students' English translation proficiency. At present, in the course of college English translation teaching, teachers mainly adopt the traditional Grammar Translation Method, which focuses on developing students' grammar knowledge. Students need to memorize a large number of words and sentences, and master all kinds of complicated rules. This method is conducive to the consolidation and improvement of students' grammar knowledge, but it is too boring. Long-term use will make students lose interest in translation, and it is not beneficial to the cultivation of translation talents. Cognitive Approach, also called Modern Grammar Translation, overcomes the drawbacks of past methods, and pays more attention to the understanding, practice and application of materials, and emphasizes translation practice in real situations. Compared with traditional grammar translation method, it is a progress. Therefore, the two are complementary to each other in the teaching of college English translation.
\end{abstract}

Keywords: Grammar Translation Method, Cognitive Approach, Translation Teaching

\section{语法翻译法和认知法在大学英语翻译教学中的应用互补性的研究}

王珂*，吴嘉平

外国语学院, 华北电力大学, 北京, 中国

邮箱

1125865328@qq.com（王珂）, jpingwu@126.com（吴嘉平）

摘要: 随着中国对外交往的扩大, 外语翻译类人才的培养也变得尤为紧迫。翻译作为对外合作和沟通的桥梁, 在我国 与世界其他国家的合作中起着举足轻重的作用。然而, 目前我国翻译人才储备仍处于青黄不接之时, 高水平的译者仍 十分缺乏。大学作为高素质人才培养的摇篮, 大学生也是国家翻译人才培养的后备军。因此, 提高大学生的英语翻译 水平显得尤为重要。当前, 在大学英语翻译教学中, 教师们主要采用的还是传统的语法翻译法。语法翻译法注重培养 学生的语法知识。而认知法又叫现代语法翻译法, 克服了以往语法翻译法的弊端, 注重对材料的理解、操练和运用, 并且强调在真实的情境下进行翻译实践, 是在传统语法翻译法基础上的进步。语法翻译法要背诵大量的单词和句型, 
掌握各种复杂的规则, 有利于学生语法知识的巩固和提高, 但却过于枯燥, 长期使用会让学生丧失对翻译的兴趣, 也 不利于翻译人才的培养。因此, 二者在大学英语翻译教学中具有很强的互补性。

关键词: 语法翻译法, 认知法, 翻译教学

\section{1. 引言}

据调查, 目前全国范围内专职翻译有四万多人, 兼职 翻译多达五十多万人, 全国共有三千多家专业的翻译公司, 但能够胜任翻译工作的人只占其中的 $10 \%$ 左右。也就是 说, 虽然有如此多的人从事翻译, 但真正高层次的人才仍 然存在很大缺口。全球化进程使中国和外国的交往不断增 多。大到国家, 小到企业、个人都需要和外界进行交往。 快速发展的经济需要和世界接轨, 日益繁荣的文化产业需 要和世界交流, 新型的企业也需要引进国外同行先进的技 术和设备. ......, 这些都离不开翻译。翻译人才处在对外 交往的前沿, 在国家对外交往中发挥重要的作用。

为了顺应这一趋势, 当前许多高校都开设了翻译硕士 专业学位为国家培养高层次的翻译人才, 弥补翻译人才的 缺口。在大学英语翻译教学中, 必须采用有效的方法 [10]。 传统的语法翻译法在训练和巩固同学们语法能力方面有 其自身优势, 但单一的使用这种方法势必让课堂变得沉闪。 而认知法是对传统语法翻译法的进步, 更加注重对学生翻 译实践能力的培养, 更好地弥补语法翻译法的弊端。

\section{2. 理论背景}

\section{1. 语法翻译法}

语法翻译法起源于拉丁语教学实践。十六世纪初, 拉 丁语成为当时世界上最普遍的语言, 被广泛地应用到政府、 学术和商业交流中。然而, 在拉丁语的消亡过程中, 它逐 渐被英语、法语和意大利语的所替代。在十九世纪的欧洲, 教育体系主要围绕教师心理学建立[4]。这一理论认为人的 身体和思想是分开的。思想由三部分组成: 意志、情感和 智力。而智力可以控制意志和情感。通过学习古典希腊和 罗马文化, 人们就可以达到这样的境界。起初, 人们认为 现代语言不利于心理学科的发展, 因而被饱受冷落。直到 十九世纪, 才被人们接受[1]。教师们仍然用教拉丁语的方 式即语法翻译法讲解现代语言。在美国, 很多高中和大学 都使用语法翻译法进行外语教学。

该方法有两个主要目标: 使学生能够阅读和翻译源语 中的文学作品; 并进一步促进其智力发展。由于很多学习 外语的人是为了能够看懂外国文学作品, 所以语法翻译法 注重培养学生的阅读和写作能力, 但其忽视了听说能力的 培养。语法翻译法是使用母语进行教学。学生首先需要学 习大量的语法规则, 之后做大量的练习巩固所学知识, 最 后逐字翻译句子。当学生的水平提高时, 就被要求翻译整 篇文章。相比翻译内容而言, 语法翻译法更加注重形式的 规范性[2]。在语法翻译法下, 学生们大都掌握了丰富的语 法规则, 基本的语言知识比较扎实, 但实践和交流能力明 显不足。而且只注重形式而忽略内容致使学生们的翻译脱
离实际, 显得比较生硬 [11]。并且大量记忆各种复杂的规 则给学生带来了很大的负担。长期下去, 学生们就会失去 对翻译的兴趣。

\section{2. 认知法}

认知法产生于二十世纪六十年代, 由美国著名心理学 家约翰·卡鲁尔提出。卡鲁尔认为学习外语主要基于学生 对语音及语法的分析和学习, 也就是对外语的掌握和控制 过程。在认知法之前, 主流的外语教学方法是听说法。但 随着科技的发展和社会的进步, 单纯机械的听说操练已经 不能满足社会发展的要求。一遍遍的操练会让同学们在保 持兴趣方面难以持之以恒。因此, 听说法逐渐被认知法所 取代。

认知法又被看作现代语法翻译法。它不仅弥补了以往 听说法的不足, 而且对传统语法翻译法来说, 也是一种进 步。认知法试图用“认知一符号”学习理论来代替“刺激一 反应”学习理论, 反对语言学习中的结构模型, 也就是不 断地重复, 机械地操练。该流派的专家认为外语学习在某 种程度上是一种有意识地创造性活动, 而不是简单地模仿, 并提倡用演绎法来讲授语法。总的来看, 认知法主要有以 下几个特点: 1) 外语教学要以学生为中心; 2) 强调有意 义的学习, 有意义的联系；3）听说读写同等重要；4）允 许学生出错；5）出错是可以理解的；6）可以采用多媒体 等形式使课堂更加生动; 7) 强调学生智力的重要性[5]。

由此可以看出, 由于认知法的这些特点, 它更能适应 社会发展的需要。认知法吸收了语言学和心理学的最新研 究成果, 从语言学、心理学以及认知科学的角度来研究教 学, 是一门比较科学的外语教学法。尤其是在大学英语翻 译教学中, 这种方法更能激发学生学习的积极性和主动性, 从而达到理想的教学效果 $[6]$ 。

\section{3. 语法翻译法和认知法在大学英语翻译教学中 的应用对比}

\section{1. 语法翻译法在大学英语翻译教学中的应用}

作为一种传统的教学方法, 语法翻译法在今天的大学 英语翻译课堂中仍然占据着重要地位。尤其是现在, 英语 课堂都属于大班教学。语法翻译法简单操作的特点使得它 仍然受到很多老师的青睐[2]。

语法翻译法在翻译教学中的应用主要表现在以下几 个方面: 1) 从教学方式看, 要求学生记忆大量语法规则, 利用有限的规则生成无限的句子, 从而实现母语和英语之 间的互译。对于很多母语为非英语的中国学生来说, 这种 方式无疑是有用的。对于欧美地区的学生来说, 一方面有 良好的语言环境; 另一方面学生人数比较少, 适合采用多 种方式进行教学。而在中国, 很多都是大班教学, 也没有 
良好的语言环境, 所以只有从语法入手, 通过进行大量的 语法训练, 掌握英语的句法规则, 从而实现英汉双语互译。 2) 从材料来源看, 要求学生大量阅读外国名著。这无疑 有助于提高学生的外国文化素养。经过长期的积累, 学生 对目的语国家的文化会有更加深刻的理解和体会, 翻译该 国语言时也会更加得心应手。众所周知, 一位优秀的翻译 不仅要有高超的句子掌控能力, 还必须有良好的文化素养, 这样才能翻译出优秀的作品。比如, 一个很简单的句子, “爱屋及乌”, 很多不懂英语国家文化的人可能会将其翻译 为“Love me, love my bird”。而真正了解文化知识的人都 知道, 在西方, 狗是人们所钟爱的动物, 被赋予很多美好 的意义。因而这个成语应该翻译为“Love me, love my $\operatorname{dog}$ ”[3]。3) 从课堂主体来看, 语法翻译法主导的课堂都 是以老师为中心。老师是课堂活动的掌控着, 而学生则是 课堂活动的被动接收者。比如, 在翻译课堂上, 老师会向 学生讲解大量的句式翻译, 学生需要不停地做笔记, 很少 提问。老师只是单方面灌输知识, 师生之间很少互动。在 课后, 老师还需要同学们做大量的翻译练习来巩固课堂上 所讲的知识。通过这样的方式, 学生对老师存在一种敬畏 心理。这会促使其努力完成自己的翻译任务, 提高自己的 翻译水平。

语法翻译法由于自身的一些优势已经在我国大学英 语翻译教学中被广泛地应用。但是在今天, 随着时代的 发展和进步, 单一地使用这种方法还是存在一些不足。 单纯的讲授语法规则会使翻译课堂变得枯燥无味, 长期 下去, 学生们会产生庆学情绪, 失去对翻译的兴趣。大 量阅读外国古典文学作品虽然会加深同学们对外国文化 的了解, 但这些作品一方面比较艰深难懂, 会打击同学 们的自信心, 另一方面由于年代久远, 应用性不强。很 多词汇句式都是古英语或中世纪英语, 现在已经不再使 用。比如在莎士比亚的文学作品中, “you” 用 “thou” 来 表示, “love”用 “loue” 来表示, “joy”用“ioy”来表 示, 还有在同一行里面, “他”既可以用 “he”, 也可以用 “hee”等等, 这些词汇由于现在不再使用, 在很大程度上 增加了理解难度。而且在以教师为中心的课堂中, 老师 就是权威。同学们没有话语权, 彼此间也无互动。翻译 本来就是一个交流的过程。如果同学们都闭门造车, 那 就不会知道和别人相比, 自己有哪些地方需要改进, 有 哪些地方还需要进一步学习。此外, 在这样的课堂中, 学生不被允许犯错, 出错就受到老师的批评, 致使学生 更加胆怯, 对翻译产生恐惧心理 [8]。

\section{2. 认知法在大学英语翻译课堂中的应用}

随着外语教学理论的不断发展, 新的教学方法层出 不穷。而认知法正是在以往教学方法的基础上产生的。 作为一种新的教学方法, 它有其自身优势, 并在当今的 大学英语翻译课堂中被广泛使用, 主要表现在以下几个 方面: 1) 从教学方式上看, 认知法把教学过程分为三个 阶段, 即理解、练习和运用。它克服了以往教学方法中 单纯的形式操练, 注重在练习之前对材料的理解, 在理 解的基础上进行联系和运用。对于翻译而言, 认知法主 张在进行翻译练习之前, 首先要对材料有一定的理解,
比如材料的题材是什么, 是属于什么类型的, 主要讲的 哪方面的知识等等, 在理解的基础上进行翻译。如果不 理解材料, 只是简单的按照句子结构逐字翻译, 译文就 会显得生涩牵强。2) 从材料来源上看, 教学材料不仅仅 局限于一些名著, 同学们可以根据自己的智力水平和理 解层次选择适合自己学习的内容。认知法把语言学习看 作是智力活动。离开了智力, 就谈不上语言学习。对于 智力差的同学来说, 如果选择那些深奥难懂的外国原著, 不仅不会提高自己的语言水平, 也会让自己产生畏难情 绪。3) 从课堂主体来看, 认知法强调以学生为中心, 培 养学生自主学习的能力。学生是课堂活动的主体。而老 师则是协助者和组织者 [9]。由传统的以老师为中心转变 为以学生为中心是现代外语教学的发展趋势。在课堂活 动中, 学生们通过自主研究和研讨得到合理的答案。在 这个过程中, 学生可以出错, 不用担心会受到惩罚。因 此, 在翻译课堂中, 老师要让学生主动学习。比如, 对 于这样一个句子 “This area, noted for its beautiful scenery, was counted as one of the eight outstanding views of Beijing”, 在讲解之前, 老师先让同学们翻译, 一个同学 的译文是“这个地方, 以美丽的景色出名, 算是北京八个 著名景点之一”。对于这个句子, 对北京文化有一定了解 的人都知道, “the eight outstanding views of Beijing” 是 “燕京八景”的意思。由于缺乏背景知识, 这位同学把这 句话按照字面意思翻译出来。在点评过程中, 老师并没 有直接说出学生翻译错了, 而是讲解有关“燕京八景”的 背景知识, 并分析了这么翻译的原因。通过这样的方式, 该同学必然会对这个知识有了更加深刻的理解和体会。

但认知法也存在一定的缺陷。认知法强调智力的重要 性[7]。对于理解力较差的学生来说, 有些时候会难以跟上 课堂进度。尤其是大班课堂中, 学生水平高低不一, 有些 时候, 老师很难做到兼顾。

\section{3. 语法翻译法和认知法的互补性}

翻译教学的目的就是为了提高学生们翻译能力。为了 更好地看出二者的互补性, 笔者对本校非英语专业 40 名本 科生进行测试。学生分成两组。二十个学生为一组。授课 内容都是相同的, 选取大学英语教材中的一段课文翻译。 老师对两组同学用不同方法进行45分钟的课堂授课。第一 组同学采用的是语法翻译法。在授课中, 老师全程进行讲 解, 比如一些重要单词、句型的翻译技巧和方法。之后布 置作业, 针对课堂所讲让学生进行翻译练习。第二组同学 采用的是认知法。在授课中, 老师用二十分钟讲解重要翻 译方法。之后, 留出五分钟时间让学生思考自己不明白或 不理解的地方。在最后的二十分钟, 老师首先让学生进行 小组讨论, 解决自己不明白或者不理解的地方。然后针对 同学们的讨论结果, 老师进行点评、引导。最后让两组同 学分别翻译一小段文章, 对其翻译能力进行测试。翻译文 本如下, In 2008, a law was passed in Switzerland which details the conditions in which any species of animals should be kept. But unlike the state of Zurich, the other 25 federal states of Switzerland has a form of direct democracy, where citizens can contest new laws or vote for changes to if there is enough collective support. 结果显示, 第二组同学的翻译 
水平比第一组同学要高, 在重点词汇、句型理解方面都要 好于第一组同学。例如, 对于第一句话, 应该要被动变主 动。在这点, 相比第一组学生, 第二组学生都能很好的进 行表达。对于重点词汇democracy、federal等, 相比第一组 同学, 第二组同学都能准确翻译出来。

从以上的分析中, 可以看出语法翻译法和认知法在外 语翻译教学中有很强的互补性。从教学方式看, 认知法克 服了语法翻译法只注重翻译形式而忽略翻译内容的缺陷, 强调对内容要有一定的理解; 从材料来源上看, 认知法认 为要根据学生的智力水平选择翻译材料。相比语法翻译法, 材料选择更加注重学生的可接受性; 从课堂主体来看, 认 知法强调以学生为中心。和语法翻译法相比, 这是一大进 步。相对于认知法的缺陷, 语法翻译法能很好地适应大班 教学, 老师对各个知识点都能做到细致讲解。无论理解力 好坏, 一般都能做到对知识的理解和接受。因此, 语法翻 译法和认知法在翻译教学中能够弥补对方的缺点, 做到取 长补短。

\section{4. 结论}

纵观外语教学法的发展历史, 没有任何一种教学方法 是十全十美的。要想达到理想的教学效果, 还是需要通过 不断的实践找到合适的方法。语法翻译法和认知法都有各 自的优点和缺点, 但却能够做到优势互补, 在当前大学英 语翻译教学中得到了广泛的应用。尤其是在当前背景下, 翻译人才的培养成为十分迫切的任务, 这两种方法的结合 会取得良好的教学效果。

\section{参考文献}

[1] 饶岩岩. 对语法翻译法在我国外语教学中适用性的思考 [J]. 乐山师范学院学报. 2008(09):(79-82)。

[2] 李丽娟. 语法翻译法在中国英语教育领域长期存在的合理 性[J]. 今日南国(理论创新版). 2008(07):(55-57)。

[3] 程春君. 浅谈语法翻译法在对外汉语教学中的运用[J]. 辽宁 师专学报(社会科学版). 2014(03):(76-77)。

[4] 高欣璐. 语法翻译法的历史、现状与启示 [J]. 品牌(下半 月). 2015(04):(121)。

[5] 李纯. 认知法在对外汉语教学中的应用 [J]. 韩山师范学院学 报. 2010(01):(100-103)。

[6] 卜时红. 认知法与外语听说强化训练刍议 [J]. 荆州师范学院 学报. 2001(06):(116-118)。

[7] 王淑娟. 认知法在英汉句子结构转换中的作用研究 [J]. 高教 研究与实践. 2010(01):(16-18)。

[8] 张迎红. 大学英语翻译教学行动研究 [J]. 现代经济信 息. 2016(18):(433)。

[9]李成静. 从“灌输”转向“建构”一论大学英语翻译教学中 教师角色的转变 $[J]$. 文史博览(理论). 2016(05):(84-85)。

[10] 薄振杰, 李和庆. 关于当前中国翻译教学研究的思考 [J]. 外 语界. 2011(05):(76-83)。

[11] 英语教学中的语法翻译法和直接法 $[\mathrm{J}]$. 段飞志. 学周 刊. 2015(33):(34)。 